\title{
Amyloid and APOE Status of Screened Subjects in the Elenbecestat MissionAD Phase 3 Program
}

\author{
C. Roberts ${ }^{1}$, J. Kaplow2 2 M. Giroux², S. Krause', M. Kanekiyo² \\ 1. Eisai Ltd., Hatfield, UK; 2. Eisai Inc., Woodcliff Lake; NJ, USA \\ Corresponding Authors: Claire Roberts, Eisai Ltd., Hatfield, UK, Email: claire_roberts@eisai.net, Phone: +44 8456761590
}

\section{Abstract}

BACKGROUND/OBJECTIVES: Elenbecestat, an oral BACE1 inhibitor that has been shown to reduce $A \beta$ levels in cerebrospinal fluid, was investigated in two global phase 3 studies in early AD. Here we report on differences observed in characteristics of APOE $\varepsilon 4$ and amyloid positive subjects in the large screening cohort.

DESIGN: Screening was performed in 5 sequential tiers over a maximum of 80 days, as part of placebo controlled, double blind phase 3 studies.

SETTING: Subjects were evaluated at sites in 7 regions (29 countries).

PARTICIPANTS: Overall, 9758 subjects were screened.

INTERVENTION: All screened subjects that were eligible received either placebo or $50 \mathrm{mg}$ QID elenbecestat post randomisation.

MEASUREMENTS: Gender, disease staging, APOE $\varepsilon 4$ status, amyloid status, amyloid positron emission tomography (PET) standard uptake value ratio (SUVr) and amyloid PET Centiloid (CL) values were determined for screened subjects; by country and region.

RESULTS: In this program, $44 \%$ of subjects were APOE $\varepsilon 4$ positive. Frequency of females was similar in both APOE $\varepsilon 4$ positive and negative groups. However, early mild AD subjects were slightly higher in the APOE $\varepsilon 4$ positive group compared with the APOE $\varepsilon 4$ negative group. $56 \%$ of subjects were amyloid positive. The mean age in the amyloid positive group was slightly higher than the amyloid negative group. The gender distribution was similar between amyloid groups. A lower number of mild cognitive impairment was observed in the amyloid positive group along with a higher number of early mild AD. APOE $\varepsilon 4$ positive subjects were higher in amyloid positive group compared to the amyloid negative group. China had the lowest APOE $\varepsilon 4$ and amyloid positivity rates with Western Europe and Oceania performing best. Subjects received florbetapir, florbetaben or flutemetamol amyloid PET tracer. Amyloid negative and positive subjects CL values were normally distributed around their respective means of $1.5 \mathrm{CL}$ and $83 \mathrm{CL}$. However, there was an appreciable overlap in the 20-40 CL range.

CONCLUSIONS: In this large cohort of cognitively impaired subjects, subject demographics characteristics were comparable regardless of APOE genotype or amyloid positivity. APOE $\varepsilon 4$ positivity and amyloid positivity varied by country and by geographical region.

Key words: MissionAD, Amyloid, APOE, elenbecestat.

\section{Introduction}

A lzheimer's disease (AD) is characterised by the $\Delta$ deposition of amyloid-beta $(\mathrm{A} \beta)$ aggregates and neurofibrillary tangles in the brain (1). The amyloid cascade hypothesis proposes that $\mathrm{A} \beta$ aggregates trigger the spreading of tau-related neurofibrillary tangles and subsequent neuronal degeneration (2). Recently, it has been shown that $\mathrm{A} \beta$ accumulation precedes tau and seems to accelerate neocortical tau pathology (3). In addition, amyloid accumulation has been shown to be poorly correlated with cognitive impairment (4), while tau accumulation shows a high correlation to neurodegeneration and loss of cognitive function (5). A $\beta$ is produced when amyloid precursor protein (APP) is cleaved sequentially by $\beta$-site APP-cleaving enzyme 1 (BACE-1; also referred to as $\beta$-secretase) and $\gamma$-secretase (6). Inhibition of BACE-1 is a potential therapeutic strategy for slowing the progression of Alzheimer's disease by reducing the production of $A \beta$.

Elenbecestat is an oral BACE-1 inhibitor that has been shown to reduce the $A \beta$ level in the cerebrospinal fluid (CSF). A $\beta$ (1-x) is a compilation of all amyloid isoforms that are cleaved by the BACE enzyme at the 1 position, that include at least the first 24 amino acids. CSF A $\beta$ (1-x) decreased by $62 \%$ compared to placebo in healthy volunteer phase 1 study (E2609-A001-002) at $50 \mathrm{mg}$; increasing to $74 \%$ at $100 \mathrm{mg}$ and $85 \%$ at $400 \mathrm{mg}$ (7). This was confirmed in patients with mild cognitive impairment $(\mathrm{MCI})$ and early mild $\mathrm{AD}$ in an elenbecestat phase 2 study (Study E2609-G000-201)[8] indicating an average of $69 \%$ decrease in CSF $A \beta(1-x)$ with $50 \mathrm{mg}$ of elenbecestat.

Elenbecestat was investigated in two global phase 3 studies (E2609-G000-301 or MissionAD1; E2609-G000-302 or MissionAD2) in early AD. The population consisted of subjects with a diagnosis of MCI due to AD and no more than $25 \%$ diagnosed as early stage mild dementia due to AD.

The studies were recommended to terminate early by the programme Data Safety Monitoring Board in September 2019, following an unfavourable benefitrisk profile of elenbecestat. At the time of termination 
approximately 959 subjects had reached 12 months of treatment (500 placebo, 459 elenbecestat). There was no evidence of potential efficacy and the adverse event profile was worse than placebo. At that time elenbecestat was the only BACE inhibitor programme ongoing, following similar outcomes with BACE inhibitor studies with verubecestat (9-10), lanabecestat (11), atabecestat (1213), and umibecestat (14).

At the time of the early termination the MissionAD studies were fully recruited with 2212 randomised subjects. This has resulted in a large cohort of 9758 screened subjects, establishing eligibility through cognitive assessments, laboratory assessments, MRI safety and amyloid status; representing a large volume of information for the Alzheimer's research community. Here we report on differences observed in characteristics of APOE $\varepsilon 4$ and amyloid positive subjects in this large screening cohort. Preliminary data was presented at AAIC and CTAD conferences during the past two years (15-17).

\section{Methods}

The MissionAD screening process was performed in 5 sequential tiers over a maximum of 80 days. Cognitive assessments, medical history and clinical diagnosis were determined in tier 1. Questionnaires were administered in tier 2 to establish baseline quality of life and to assess any suicidality risk. Laboratory assessments, including APOE $\varepsilon 4$ status, were conducted in tier 3. An MRI scan was done at tier 4 and finally an amyloid PET scan or a CSF sample was taken to determine amyloid status in tier 5 .

\section{Amyloid PET Scans}

Three amyloid PET tracers were used for amyloid assessment depending on availability of the tracer manufacturing facilities: florbetapir (Amyvid ${ }^{\mathrm{TM}}$ ), florbetaben (Neuraceq ${ }^{\mathrm{TM}}$ ) or flutemetamol (Vizamyl ${ }^{\mathrm{TM}}$ ). Florbetaben was prioritised if more than one tracer was available at an imaging facility. Florbetaben and flutemetamol emission acquisitions require a 20-minute scan, 90-110-minute post injection. Florbetapir emission acquisition requires a 20 minute scan, 50-70 minutes post injection.

Amyloid PET status was assessed centrally by visual read, by a radiologist blinded to cognitive status. The label of each tracer defines the number of positive regions to claim positivity on a visual read. All scans were analysed by readers trained on the guidelines established by the manufacturer. Reading for visual positivity includes the following regions for analysis:

- Florbetapir (Amyvid ${ }^{\mathrm{TM}}$ ) Frontal cortex (excluding midline), medial frontal cortex (including Anterior cingulate), parietal cortex (excluding midline), medial parietal cortex (precuneus and/or posterior cingulate), temporal cortex, and occipital cortex.

- Florbetaben (Neuraceq ${ }^{\mathrm{TM}}$ ) Lateral temporal, frontal lobes, posterior cingulate/precuneus, parietal lobes.

- Flutemetamol (Vizamyl ${ }^{\mathrm{TM}}$ ) Frontal lobes (axial \& sagittal views), posterior cingulate and precuneus (sagittal \& coronal views), temporal lobes - lateral regions (axial views - coronal views as supportive), parietal lobes - lateral regions (coronal views - axial views as supportive), striatum (axial views - sagittal views as supportive).

Amyloid PET SUVr data was calculated as mean composite SUVr (a simple average of cingulate, frontal, parietal, and temporal corticies) and mean composite SUVr including occipital region (a simple average of cingulate, frontal, parietal, temporal, and occipital corticies) using whole cerebellum as reference region.

In addition, amyloid PET data was combined across the three tracers using the Centiloid methodology for the mean composite SUVr. The Centiloid project has driven the creation of a 100-point scale termed "Centiloid (CL)," which is an average value of zero in "high certainty" amyloid negative subjects and an average of 100 in "typical" AD patients (Klunk et al., 2015; http:/ / www. gaain.org/ centiloid-project).

\section{Centiloid Conversion}

The following equations were used to calculate the mean composite SUVr in Centiloid for each tracer as derived by Bioclinica $(18,19)$ : Florbetapir: 205.72 * mean composite SUVr - 209.63; Florbetaben: 175.57 * mean composite SUVr - 173.21; Flutemetamol: 145.58 * mean composite SUVr - 139.29. All tracers: Mean composite SUVr with whole cerebellum region used as reference region is used.

\section{CSF Amyloid Analysis}

CSF sample collection was an option in this study, to determine baseline amyloid burden status. This could replace or be in addition to a baseline amyloid scan. During the study two platforms were utilized for analysis; $A \beta(1-42)<250 \mathrm{pg} / \mathrm{mL}$ from Alzbio3 run at the ADNI core lab (Dr. Leslie Shaw) and the Lumipulse ${ }^{\mathrm{TM}}$ platform from Fujirebio, using a total tau: $\mathrm{A} \beta(1-42)$ ratio greater than 0.37 to indicate positive amyloid status. The Lumipulse CSF cutpoint was established (20); note: $>0.37$ ratio was set prior to the incorporation of the issued IRMM A $\beta(1-42)$ reference standard.

\section{APOE Status Determination}

APOE genotyping was performed using a real time PCR Taqman assay, developed and performance determined by Brooks Life Sciences. The subjects were categorized into APOE $\varepsilon 4$ positive if they had at least one $\varepsilon 4$ allele, and negative if they did not have an $\varepsilon 4$ allele. 
Table 1. Recruitment status

\section{Final dataset}

Total screened subjects

Screened subjects with known APOE status (tier 3)

Screened subjects with known amyloid status, PET or CSF (tier 5)

Screened subjects with known APOE \& amyloid status (PET or CSF)

Screened subjects with known PET status and available SUVr data

Randomised subjects

\begin{tabular}{|c|c|}
\hline Number of Subjects & Percentage \\
\hline 9758 & $100 \%$ \\
\hline 5710 & $58.5 \%$ \\
\hline 4121 & $42.2 \%$ \\
\hline 4077 & $41.8 \%$ \\
\hline 3492 & $35.8 \%$ \\
\hline 2212 & $22.7 \%$ \\
\hline
\end{tabular}

Table 2. Demography of APOE $\varepsilon 4$ and amyloid positive and negative screened subjects

\begin{tabular}{|c|c|c|c|c|c|}
\hline & \multicolumn{2}{|c|}{$\begin{array}{l}\text { Screened subjects with known } \\
\text { APOE status (tier } 3 \text { ) }\end{array}$} & \multicolumn{2}{|c|}{$\begin{array}{l}\text { Screened subjects with known } \\
\text { APOE status \& amyloid status (PET or CSF) }\end{array}$} & \multirow{2}{*}{$\begin{array}{l}\text { Screened subjects with } \\
\text { known amyloid PET } \\
\text { status and SUVr data }\end{array}$} \\
\hline & APOE $\varepsilon 4$ Positive & APOE $\varepsilon 4$ Negative & Amyloid Positive & Amyloid Negative & \\
\hline Subjects, $\mathrm{n}(\%)$ & $2535(44)^{*}$ & $3175(56)^{*}$ & $2299(56)^{* \star}$ & $1778(44)^{* *}$ & $3492(100)$ \\
\hline Age, year (range) & $71(50-85)$ & $71(50-85)$ & $72(50-85)$ & $69(50-85)$ & $70(50-85)$ \\
\hline \multicolumn{6}{|l|}{ Gender, n (\%) } \\
\hline Female & $1282(51)$ & $1642(52)$ & $1178(51)$ & $925(52)$ & $1816(52)$ \\
\hline MCI & $2052(81)$ & $2661(84)$ & $1925(84)$ & $1599(90)$ & $3028(87)$ \\
\hline Early mild AD & $369(15)$ & $322(10)$ & $368(16)$ & $140(8)$ & $420(12)$ \\
\hline Other/missing & $114(4)$ & $192(6)$ & $6(<1)$ & $39(2)$ & $44(1)$ \\
\hline \multicolumn{6}{|c|}{ APOE $\varepsilon 4$ Status, $\mathrm{n}(\%)$} \\
\hline APOE $\varepsilon 4$ positive & $2535(100)$ & 0 & $1472(64)$ & $397(22)$ & $1563(45)$ \\
\hline Negative & $393(23)$ & $1354(65)$ & $56(3)$ & $1692(100)$ & $1652(47)$ \\
\hline
\end{tabular}

${ }^{*}$ The percentages are based on the subjects with ApoE4 status. **The percentage are based on the subjects with amyloid status (PET or CSF). Other percentages are based on each group.

\section{Results}

Overall, 9758 subjects were screened in MissionAD, of which 5710 had a known APOE status, 4121 had a known amyloid status (PET or CSF), 4077 had a known APOE and amyloid status, 3492 had a known amyloid status and available SUVr data, and 2212 subjects were randomised (Table 1). The majority of subjects, that reached tiers 3-5, had a MMSE score $\geq 24$, CDR Global score of 0.5 , and a cognitive impairment of $\geq 1$ standard deviation from age-adjusted norms in the International Shopping List Task.

\section{APOE Genotype}

Of the 5,710 screened subjects with known APOE genotype, $44 \%$ were APOE $\varepsilon 4$ positive (Table 2). The mean age in both the APOE groups was 71 years. Frequency of females was similar in both APOE $\varepsilon 4$ positive and negative groups $(51 \%$ and $52 \%)$. Early mild AD subjects were slightly higher in the APOE $\varepsilon 4$ positive group compared with the APOE $\varepsilon 4$ negative group (15\% and $10 \%)$.
Figure 1. APOE and amyloid status by region (Dotted red lines indicate regional means)

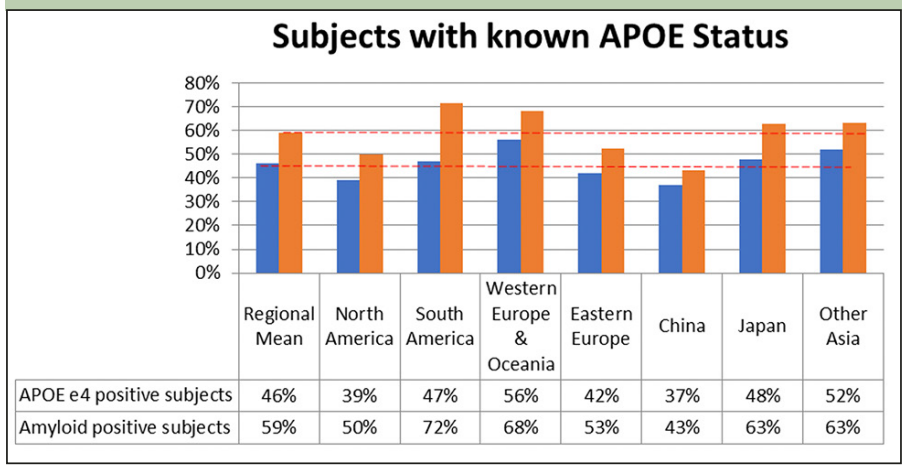

\section{Amyloid Status}

Of the 4,077 screened subjects with known APOE and amyloid status (as determined either by PET or CSF), $56 \%$ were amyloid positive (Table 2). The mean age in the amyloid positive group was slightly higher (72 years c.f. 69 years). The gender distribution was similar between amyloid groups. A lower number of $\mathrm{MCI}$ was observed in the amyloid positive group ( $84 \%$ and $90 \%)$ along with a 
higher number of early mild AD (16\% and $8 \%)$. APOE $\varepsilon 4$ positive subjects were higher in amyloid positive group compared to the amyloid negative group (64\% and $22 \%$ ).

Figure 2. Distribution of mean composite Centiloid for amyloid negative and positive subjects



\section{APOE and Amyloid Status Varies Depending on Region $\mathcal{E}$ Country}

29 countries spanning the 7 regions of North America (Canada, USA), South America (Argentina, Chile, Mexico), Western Europe \& Oceania (Australia, Austria, Denmark, Finland, France, Germany, Greece, Italy, Portugal, South Africa, Spain, UK), Eastern Europe (Bulgaria, Croatia, Czech Republic, Hungary, Poland, Russia, Slovakia), China, Japan, and Other Asia (Singapore, South Korea, Taiwan) participated in the studies.

Mean APOE $\varepsilon 4$ positivity across the countries was $48 \%$. This was lowest in Mexico $<$ Taiwan $<$ Greece $<$ Bulgaria $<$ Slovakia, but care needs to be used where numbers are too low to interpret. The highest rate was in France $>$ Australia $>$ Finland $>$ Hungary $>$ South Africa (Table 3). Mean amyloid positivity was $64 \%$ across the countries. This was lowest in Singapore $<$ Croatia $<$ Poland $<$ China $<$ Taiwan and highest in Greece $>$ France $>$ Italy $>$ Hungary $>$ Australia (Table 3). Grouping the countries into regions reduced variability. The mean APOE $\varepsilon 4$ positivity and amyloid positivity across the regions was $46 \%$ and $59 \%$, respectively. China had the lowest APOE $\varepsilon 4$ and amyloid positivity rates, while Western Europe and Oceania the highest rates (Figure 1).

\section{Amyloid PET Status}

$53 \%$ of Subjects screened with a known amyloid status and amyloid PET SUVr data were amyloid positive. Overall, $1563(45 \%)$ subjects were APOE $\varepsilon 4$ positive, 1816 $(52 \%)$ were female, and $3028(87 \%)$ were $\mathrm{MCI}$ and 420 $(12 \%)$ mild early AD (Table 2$)$.

\section{Amyloid PET SUVr}

In total, 386, 2548 and 558 subjects received florbetapir, florbetaben or flutemetamol amyloid PET tracer, of which $218(56 \%), 1292(51 \%)$ and $330(59 \%)$ were determined visually to be amyloid positive, respectively. Amyloid PET mean composite (SD; min - max) SUVr for amyloid negative subjects were $1.03(0.11 ; 0.80-1.48), 0.99(0.08$; $0.70-1.45)$ and $0.98(0.11 ; 0.80-1.38)$ for florbetapir, florbetaben or flutemetamol, respectively. Amyloid PET mean composite (SD; min - max) SUVr for amyloid positive subjects were $1.41(0.18 ; 0.94-1.94), 1.47(0.22$; $0.80-2.22)$ and $1.49(0.19 ; 0.95-1.99)$ for florbetapir, florbetaben or flutemetamol, respectively. Including the occipital region in the mean composite SUVR had very little impact on the data.

\section{Amyloid PET Conversion to Centiloid Mean Composite}

Centiloid (CL) conversion, combining data for the 3 tracers, calculated a mean (SD) of 1.5 (15.2) CL for amyloid negative subjects $(\mathrm{n}=1652)$ and 82.7 (36.7) CL for amyloid positive $(n=1840)$. No differences were observed in the individual CL values between the tracers.

\section{Mean Composite Centiloid Distribution}

Amyloid negative and positive subjects CL values were normally distributed around their respective means of $1.5 \mathrm{CL}$ and $83 \mathrm{CL}$. However, there was an appreciable overlap in the 20-40 CL range; minimum and maximum values of -50 and $95 \mathrm{CL}$ for amyloid negative and 33 and $217 \mathrm{CL}$ for amyloid positive subjects (Figure2).

There was no obvious impact of gender on the distribution of CL, with mean (SD) composite of 85 (35.6) CL and 81 (37.8) CL for amyloid positive, and 3.2 (16.1) $\mathrm{CL}$ and 0 (14.0) CL for amyloid negative, for females and males respectively.

More amyloid positive subjects were APOE $\varepsilon 4$ positive $(65 \%)$ compared to APOE $\varepsilon 4$ negative $(35 \%)$, with the mean 10 CL higher in APOE $\varepsilon 4$ positive ( $86 \mathrm{CL}$, SD 34.2) than APOE $\varepsilon 4$ negative (76 CL, SD 40.4) in the amyloid positive population. A majority $(77 \%)$ of the APOE $\varepsilon 4$ positive subjects were also amyloid positive.

Of the 420 mild early AD subjects $69 \%$ were amyloid positive, while in the $3028 \mathrm{MCI}$ subjects $51 \%$ were amyloid positive. There was a shift in the mean by approximately $9 \mathrm{CL}$ in the amyloid positive cohort, $81 \mathrm{CL}$ (SD 36.6) for MCI subjects and 90 CL (SD 36.8) for mild early AD subjects.

\section{Discussion}

Alzheimer's Disease clinical trials, due to the complexity required to identify the correct population, include many screening assessments that encompass not 


\section{Table 3. APOE and amyloid status by country}

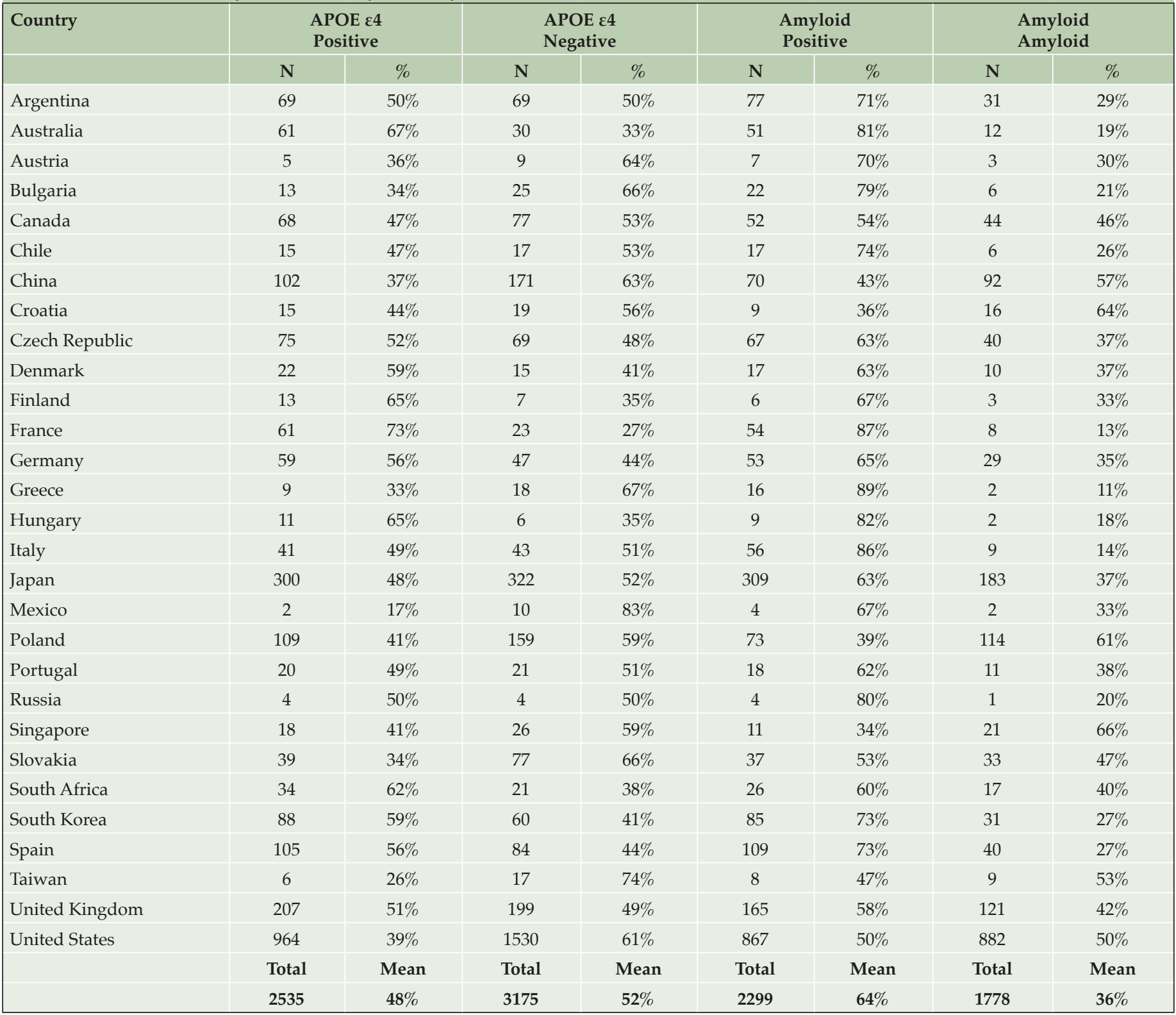

only traditional elements but also multiple cognitive, biomarker and imaging assessments. Especially in large global studies, there is an expectation of a high screen failure rate, while tiered screening is often required in order to reduce unnecessary burden on subjects and control costs.

For MissionAD the screening approach was to conduct the cognitive assessments and study specific clinical diagnosis in the first tier to eliminate the highest proportion of subjects not suitable for the trial. Approximately $41 \%$ of subjects screen failed due to cognitive or suicidality assessments. About $17 \%$ further subjects failed due to lab assessments. Finally, in the last tier a further $19 \%$ of subjects screen failed when amyloid status was determined to be negative. Overall, the screen fail rate in the early $\mathrm{AD}$ population (MCI and early mild AD) of MissionAD was $77 \%$. This screen failure rate is in the same range as reported in studies with similar populations. For example, the verubecestat APECS study in prodromal AD had a SF rate of $68 \%$ (10), the AMARANTH and DAYBREAK studies with lanabecestat in early and mild AD had SF rates of $68 \%$ and $70 \%$, respectively (11). The slightly higher rate in MissionAD may be explained by the focus on recruiting a higher proportion of MCI subjects ( $>75 \%$ ) in this program.

When assessed at the end of the screening cascade, amyloid positivity (amyloid PET visual read or amyloid $\mathrm{CSF}$ ) was a global average of $56 \%$. Of note, the positivity rates varied from country to country. It is important to understand where rates may be low, so that recruitment enrichment strategies can be employed in those areas. Countries where the positivity rates were low may be reflective of populations that more readily report subjective cognitive decline or the "worried well", 
lack of access to technology, or lack of access to care. Socioeconomic status and different healthcare systems may result in a greater focus on subjective cognitive symptoms. Lowest rates of positivity in MissionAD were observed in North America and China. APOE $\varepsilon 4$ positivity also varied by country and was lowest in North America and China. Summation of these components supported amyloid positivity as highly correlated with APOE $\varepsilon 4$ positivity.

These data reinforce the need to be aware of the variability in APOE $\varepsilon 4$ and amyloid positivity when designing clinical trials, and the importance of having strategies available to mitigate. Various approaches were employed in this trial to maximise eligible subjects, however, the screen failure rate for MissionAD was still 77\% (2212 randomised out of the 9758 screened subjects).

This study allowed the use of three different amyloid PET tracers with florbetaben being used in $75 \%$ of the subjects in this study. We presented the SUVr data as a mean composite with and without the occipital region for all tracers. However, it should be noted that per the manufacturers label the occipital region is appropriate only for florbetaben. Data indicate that the tracers were comparable and could be grouped for comparisons by using the Centiloid methodology supporting the use of multiple tracers in a global trial to expand countries able to participate.

Amyloid negative and positive subjects Centiloid values were normally distributed with an appreciable overlap in the 20-40 CL range. Distribution was shifted to higher Centiloid values by APOE $\varepsilon 4$ positive status and mild early AD diagnosis. Similar results have been shown by other groups when examining large cohort studies (21) and have revealed similar, bimodal distributions of $\mathrm{CL}$ in the population studied (22). These results reveal similar factors influenced amyloid positivity as was evident with amyloid PET visual read.

In this large cohort of cognitively impaired subjects, just under half were positive for APOE $\varepsilon 4$ and just over half were amyloid positive. APOE $\varepsilon 4$ positivity and amyloid positivity varied by country and by geographical region. Subject demographics characteristics were comparable regardless of APOE genotype or amyloid positivity. A higher APOE $\varepsilon 4$ or amyloid positivity rate generally reflected a higher identification of early AD subjects in most regions. APOE $\varepsilon 4$ positive subjects are more likely to have elevated amyloid.

Acknowledgments: Study participants and all sites that took part in MissionAD. Editorial support provided by JD Cox, PhD and Mayville Medical Communications.

Funding: Funding for the studies, analyses, and editorial support was provided by Eisai Inc.

\section{Disclosures: All authors are, or were, employees of Eisai Inc or Eisai Ltd.}

Ethical standards: The study was performed in full compliance with International Council for Harmonisation of Technical Requirements for Pharmaceuticals for Human Use and all applicable local Good Clinical Practice and regulations.
Open Access: This article is distributed under the terms of the Creative Commons Attribution 4.0 International License (http:/ / creativecommons.org/ licenses/by/4.0/), which permits use, duplication, adaptation, distribution and reproduction in any medium or format, as long as you give appropriate credit to the original author(s) and the source, provide a link to the Creative Commons license and indicate if changes were made.

\section{References}

1. Masters CL, Bateman R, Blennow K, Rowe CC, Sperling RA, Cummings JL. Alzheimer's disease. Nat Rev Dis Primers 2015;1:15056.

2. Selkoe DJ, Hardy J. The amyloid hypothesis of Alzheimer's disease at 25 years. EMBO Mol Med 2016;8:595-608.

3. Hanseeuw BJ, Betensky RA, Jacobs HI, et al. Association of Amyloid and Tau With Cognition in Preclinical Alzheimer Disease: A Longitudinal Study. JAMA Neurol. 2019;76(8):915-924.

4. Jack CR, Knopman DS, Jagust WJ, et al. Hypothetical model of dynamic biomarkers of the Alzheimer's pathological cascade. Lancet Neurol. 2010;9(1):119-28.

5. Duyckaerts C, Brion JP, Hauw JJ, Flament-Durand J. Quantitative assessment of the density of neurofibrillary tangles and senile plaques in senile dementia of the Alzheimer type. Comparison of immunocytochemistry with a specific antibody and Bodian's protargol method. Acta Neuropathol. 1987;73(2) :167170.

6. Yan R, Vassar R. Targeting the $\beta$ secretase BACE1 for Alzheimer's disease therapy. Lancet Neurolog. $2014 ; 13: 319-29$.

7. Albala B, Kaplow J, Lai R, Matijevic M, Aluri J, Satlin A. CSF Amyloid Lowering in Human Volunteers after 14 days Oral Administration of the Novel BACE1 Inhibitor E2609. 2012. AAIC abstract published July 2012 (oral presentation).

8. Majid O, Gee M, Albala B, et al. Dose-related reductions of CSF amyloid $\beta$ (1-x) by E2609, a novel BACE1 inhibitor, in patients with mild cognitive impairment due to Alzheimer's disease (AD) and mild-to-moderate AD dementia. CTAD 2016;San Diego: P3-28 (poster presentation).

9. Egan MF, Kost J, Tairot PN et al. Randomised trial of verubecestat for mild-tomoderate Alzheimer's disease. N. Eng. J. Med. 2018;378(18): 1691-1703.

10. Egan MF, Kost J, Voss T, et al. Randomized Trial of Verubecestat for Prodromal Alzheimer's Disease. N Engl J Med. 2019;380(15):1408-1420.

11. Wessels AM, Tariot PN, Zimmer JA, et al. Efficacy and Safety of Lanabecestat for Treatment of Early and Mild Alzheimer Disease: The AMARANTH and DAYBREAK-ALZ Randomized Clinical Trials. JAMA Neurol. 2020 Feb 1;77(2):199-209.

12. Henley D, Raghavan N, Sperling R, Aisen P, Raman R, Romano G. Preliminary Results of a Trial of Atabecestat in Preclinical Alzheimer's Disease. N Engl J Med. 2019;380(15):1483-1485.

13. Novak G, Streffer JR, Timmers M, et al. Long-term safety and tolerability of atabecestat (JNJ-54861911), an oral BACE1 inhibitor, in early Alzheimer's disease spectrum patients: a randomized, double-blind, placebo-controlled study and a two-period extension study. Alzheimers Res Ther. 2020;12(1):58.

14. Graf A, Borowsky B, Tariot P, et al. Alzheimer's Prevention Initiative Generation Program: Update And Next Steps. 2019. CTAD abstract [OC11].

15. Roberts C, Kaplow J, Giroux M, Krause S, Kanekiyo M. Amyloid burden assessed by three amyloid PET tracers in the elenbecestat MissionAD phase 3 program. 2020. AAIC abstract published, July 2020 (poster presentation).

16. Roberts C, Kanekiyo M, Albala B. Amyloid positive subject characteristics in the elenbecestat MissionAD phase 3 program. 2019. CTAD abstract published, December 2019 (poster presentation).

17. Roberts C, Kanekiyo M, Kaplow J, Albala B. APOE4 status and amyloid burden differences across regions in the elenbecestat MissionAD phase 3 program. 2019. AAIC abstract published, July 2019 (poster presentation).

18. Klunk WE, Koeppe RA, Price JC, et al. The Centiloid Project: standardizing quantitative amyloid plaque estimation by PET. Alzheimers Dementia. 2015;11(1):1-15

19. Adamczuk K, Sampat M, Bracoud L, Runkle M, Gorman B, Scott D. Centiloid scale in practice: Effects of different SUVr reference regions and comparison of centiloid cut-offs. Alzheimer's \& Dementia. 2019;15(7) suppl: 223-224.

20. Kaplow J, Vandijck M, Gray J, et al. Concordance of Lumipulse cerebrospinalfluid t-tau/A $\beta 42$ ratio with amyloid PET status. Alzheimer'sDement. 2020;16:144-152.

21. Mormino E, Betensky RA, Hedden T, et al. Amyloid and APOE $\varepsilon 4$ interact to influence short-term decline in preclinical Alzheimer's disease. Neurology. 2014;82:1760-1867.

22. La Joie R, Ayakta N, Seeley WW, Borys E et al. Multisite study of the relationships between antemortem [11C]PiB-PET Centiloid values and postmortem measures of Alzheimer's disease neuropathology. Alzheimer's \& Dementia. 2018; 1-12. 